\title{
INVERSE PARTICIPATION RATIOS IN THE XX SPIN CHAIN
}

\author{
EMMANUEL TSUKERMAN
}

\begin{abstract}
We continue the study of the Inverse Participation Ratios (IPRs) of the XXZ Heisenberg spin chain initiated by Misguich, Pasquier and Luck (2016) by focusing on the case of the XX Heisenberg Spin Chain. For the ground state, Misguich et al. note that calculating the IPR is equivalent to Dyson's constant term ex-conjecture. We express the IPRs of excited states as an apparently new "discrete" Hall inner product. We analyze this inner product using the theory of symmetric functions (Jack polynomials, Schur polynomials, the standard Hall inner product and $\omega_{q, t}$ ) to determine some exact expressions and asymptotics for IPRs. We show that IPRs can be indexed by partitions, and asymptotically the IPR of a partition is equal to that of the conjugate partition. We relate the IPRs to two other models from physics, namely, the circular symplectic ensemble of Dyson and the DysonGaudin two-dimensional Coulomb lattice gas. Finally, we provide a description of the IPRs in terms of a signed count of diagonals of permutohedra.
\end{abstract}

\section{INTRODUCTION}

Recently in [MPL16, Misguich, Pasquier and Luck began a numerical study of the so-called Inverse Participation Ratios (IPRs) in the spin-1/2 XXZ chains. IPRs give a measure of localization of the system with respect to a preferential basis. Roughly speaking, large IPRs imply that the system will be found in a single state, whereas small IPRs imply that the system is equally likely to be found in any available state. The interest in the question stems from the fact that integrable systems generally fail to reach thermal states. Misguich et al. ask in particular about the IPRs in the special case of the XX spin chain with respect to the Ising basis.

We let $L$ denote the length of the spin chain and restrict attention to the space spanned by Ising bases of $M$ down spins. To analyze the IPRs, we will proceed as follows. First, we will employ the Bethe ansatz to obtain an expression for the eigenstates in terms of the Ising basis. We will see then that the IPRs are naturally labeled by partitions, and are equal to

$$
\mathbf{t}_{\lambda}=\left(M ! L^{2 M}\right)^{-1} \sum_{\theta_{1}, \ldots, \theta_{M} \in\left\{0, \frac{2 \pi}{L}, \ldots, \frac{2 \pi(L-1)}{L}\right\}}\left|s_{\lambda}\left(e^{i \theta_{1}}, \ldots, e^{i \theta_{M}}\right)\right|^{4}\left|\prod_{i<j}\right| e^{i \theta_{i}}-\left.e^{i \theta_{j}}\right|^{4},
$$

where $s_{\lambda}$ is the Schur polynomial (a specialization of the Jack polynomials). This will naturally lead us to the theory of symmetric functions. We will analyze these quantities in complete mathematical rigor (section 5 and on).

Before employing techniques from symmetric function theory, we provide several physical interpretations of the IPRs and discuss the ground state. The calculation of the ground state IPR $\left(s_{\lambda}=1\right)$ is equivalent to Dyson's constant term ex-conjecture, an important motivation for much of the development of the theory of Macdonald polynomials. For excited states, we can interpret the IPR as the expected value of $\left|s_{\lambda}\right|^{4}$ over the Dyson-Gaudin two-dimensional Coulomb lattice gas. In the limit $L \rightarrow \infty$ the gas is no longer restricted to a lattice. In this situation, the IPR can be interpreted as the expected value of $\left|s_{\lambda}\right|^{4}$ over the circular symplectic ensemble (CSE) of Dyson. The CSE is a modification of the Gaussian symplectic ensemble (GSE), a key matrix ensemble in random matrix theory.

Next we study the IPRs using the tools of symmetric function theory, namely, Jack polynomials, Hall inner product and $\omega_{q, t}$. We interpret the IPRs in terms of a "discrete" Hall inner product and show that assuming, roughly, $L>2 M$ the discrete Hall inner product and the traditional one coincide.

Date: March 7, 2018. 
This allows us to use the orthogonality of the Jack polynomials to evaluate the IPRs in terms of the transition coefficients from Schur polynomials to Jack polynomials. When these are known, we are able to determine exact expressions for the IPRs and their asymptotics. Finally, we show that curiously, if $\lambda^{\prime}$ is the conjugate partition of $\lambda$, then

$$
\mathbf{t}_{\lambda}=\mathbf{t}_{\lambda^{\prime}}
$$

assuming, roughly, that $L>2 M \gg 1$.

\section{Contents}

1. Introduction

2. Inverse Participation Ratios

3. Coordinate Bethe ansatz for the periodic XX Heisenberg model

4. Connection between the IPRs of XX, the circular symplectic ensemble and the Dyson-Gaudin Coulomb gas

4.1. Circular symplectic ensemble

4.2. Dyson-Gaudin Coulomb gas

5. Jack and Schur polynomials

5.1. Partitions

5.2. Jack polynomials

5.3. Schur polynomials

5.4. Transition matrices

6. Calculating the IPRs of XX

6.1. IPRs and diagonals of permutahedra

7. Future Directions

References

\section{Inverse Participation Ratios}

In this section we provide the basic definition of the Inverse Participation Ratios ([ET72], [MPL16]).

Definition 1. Let the normalized eigenvectors of a hamiltonian $\mathcal{H}$ be $\left\{\left|\psi_{i}\right\rangle\right\}_{i=1, \ldots, D}$ and assume they are non-degenerate. The inverse participation ratio (IPR) of an eigenstate $\left|\psi_{k}\right\rangle$ in a preferential basis $\left\{\left|a_{i}\right\rangle\right\}_{i=1, \ldots, D}$ is

$$
\mathbf{t}_{k}:=\sum_{i=1}^{D}\left|\left\langle a_{i} \mid \psi_{k}\right\rangle\right|^{4} .
$$

The maximum value of this quantity is reached when an eigenstate coincides with a single basis state, in which case $t_{\max }=1$. The minimum value is reached for eigenstates which are uniform superpositions of all the basis states, with the same modulus $\left|\left\langle a_{i} \mid \psi_{k}\right\rangle\right|=\frac{1}{\sqrt{D}}$. This maximally delocalized limit gives $t_{\min }=\frac{1}{D}$.

We note that if

$$
\tilde{\psi}_{k}=\sum_{i} c_{i}\left|a_{i}\right\rangle
$$

is an unnormalized multiple of $\psi_{k}$, then

$$
\left|\left\langle a_{i} \mid \psi_{k}\right\rangle\right|^{4}=\frac{\left|c_{i}\right|^{4}}{\left(\sum_{j}\left|c_{j}\right|^{2}\right)^{2}}
$$


and

$$
\mathbf{t}_{k}=\frac{\sum_{i}\left|c_{i}\right|^{4}}{\left(\sum_{j}\left|c_{j}\right|^{2}\right)^{2}}
$$

\section{Coordinate Bethe ansatz for the Periodic XX Heisenberg model}

We consider the XX spin chain (XXZ with anisotropy parameter $\Delta=0$ ) with periodic boundary conditions and $L$ sites. The Hamiltonian is given by

$$
\mathcal{H}=\sum_{i=1}^{L} S_{i}^{x} S_{i+1}^{x}+S_{i}^{y} S_{i+1}^{y} .
$$

We will single out the preferential basis called the Ising configuration for the IPRs. These are the eigenstates of all $S_{i}^{z}$ :

$$
|\uparrow \uparrow \downarrow \cdots\rangle,|\uparrow \downarrow \uparrow \cdots\rangle, \ldots
$$

We restrict attention to the subspace spanned by Ising configurations having $M$ down spins. This space has dimension $\left(\begin{array}{c}L \\ M\end{array}\right)$. The coordinate Bethe ansatz tells us that the wavenumbers $k_{j}, j=1, \ldots, M$, and Bethe roots $I_{j}, j=1, \ldots, M$, satisfy

$$
L k_{j}=2 \pi I_{j}, \quad j=1,2, \ldots, M .
$$

For simplicity, we will be assuming that $M$ is odd, so that the $I_{j}$ are in $\{0,1, \ldots, L-1\} \bmod L$ [ŚB13]. All statements can be adapted to the case of even $M$, in which case the $I_{j}$ are half integers. To avoid the nullity of the wavefunction, the wavenumbers must be distinct.

We use the shorthand notation

$$
|\mathbf{x}\rangle=\left|x_{1} \cdots x_{M}\right\rangle, \quad x_{1}<x_{2}<\cdots<x_{M}
$$

for the Ising basis with down spins at $x_{1}, \ldots, x_{M}$ and

$$
\mathbf{k}=\left(k_{1}, \ldots, k_{M}\right) \text {. }
$$

The eigenvectors of $\mathcal{H}$ are now given by

$$
\psi_{\mathbf{k}}=\sum_{\mathbf{x}} c(\mathbf{x})|\mathbf{x}\rangle=\sum_{\mathbf{x}} \sum_{\pi \in S_{M}} \operatorname{sgn}(\pi) e^{i \pi(\mathbf{k}) \cdot \mathbf{x}}|\mathbf{x}\rangle=\sum_{\mathbf{x}} \operatorname{det}\left(e^{i k_{a} x_{b}}\right)_{a, b}|\mathbf{x}\rangle .
$$

By (6), the IPRs are equal to

$$
\mathbf{t}_{k}=\frac{\sum_{\mathbf{x}}\left|\operatorname{det}\left(e^{i k_{a} x_{b}}\right)_{a, b}\right|^{4}}{\left(\sum_{\mathbf{x}}\left|\operatorname{det}\left(e^{i k_{a} x_{b}}\right)_{a, b}\right|^{2}\right)^{2}}
$$

The denominator of (13) is relatively easy to evaluate. We have

$$
\begin{gathered}
|c(\mathbf{x})|^{2}=c(\mathbf{x}) \bar{c}(\mathbf{x})=\left(\sum_{P \in S_{M}} \operatorname{sgn}(P) e^{i P(\mathbf{k}) \cdot \mathbf{x}}\right)\left(\sum_{Q \in S_{M}} \operatorname{sgn}(Q) e^{-i Q(\mathbf{k}) \cdot \mathbf{x}}\right)= \\
=\sum_{P, Q \in S_{M}} \operatorname{sgn}(P) \operatorname{sgn}(Q) e^{i(P(\mathbf{k})-Q(\mathbf{k})) \cdot \mathbf{x} .}
\end{gathered}
$$

We now use the fact that the determinant is zero whenever any two of $x_{i}$ are equal. This allows us to remove the restriction of the $x_{i}$ being distinct. In addition, we must divide by $M$ ! to account for the order. We also note that

$$
\sum_{x_{1}, \ldots, x_{M}=1}^{L} \prod_{j=1}^{M} e^{i x_{j} m_{j}}=\prod_{j=1}^{M} \sum_{x_{j}=1}^{L} e^{i x_{j} m_{j}} .
$$


We have

$$
\begin{gathered}
\sum_{\mathbf{x}}|c(\mathbf{x})|^{2}=\frac{1}{M !} \sum_{x_{1}, \ldots, x_{M}=1}^{L}|c(\mathbf{x})|^{2}=\frac{1}{M !} \sum_{x_{1}, \ldots, x_{M}=1}^{L} \sum_{P, Q \in S_{M}} \operatorname{sgn}(P) \operatorname{sgn}(Q) \prod_{j=1}^{M} e^{i x_{j}\left(k_{P(j)}-k_{Q(j)}\right)} \\
=\frac{1}{M !} \sum_{P, Q \in S_{M}} \operatorname{sgn}(P) \operatorname{sgn}(Q) \sum_{x_{1}, \ldots, x_{M}=1}^{L} \prod_{j=1}^{M} e^{i x_{j}\left(k_{P(j)}-k_{Q(j)}\right)}= \\
=\frac{1}{M !} \sum_{P, Q \in S_{M}} \operatorname{sgn}(P) \operatorname{sgn}(Q) \prod_{j=1}^{M} \sum_{x_{j}=1}^{L} e^{i x_{j}\left(k_{P(j)}-k_{Q(j)}\right)} .
\end{gathered}
$$

Now $k_{j}=\frac{2 \pi I_{j}}{L}$ and $0<\left|I_{j}-I_{i}\right|<L$. Consequently,

$$
\sum_{x_{j}=1}^{L} e^{i x_{j}\left(k_{P(j)}-k_{Q(j)}\right)}=L \delta\left(k_{P(j)}=k_{Q(j)}\right) .
$$

Substituting,

$$
\sum_{\mathbf{x}}|c(\mathbf{x})|^{2}=L^{M} \frac{1}{M !} \sum_{P, Q \in S_{M}} \operatorname{sgn}(P) \operatorname{sgn}(Q) \prod_{j=1}^{M} \delta\left(k_{P(j)}=k_{Q(j)}\right)=L^{M} .
$$

Therefore

$$
\mathbf{t}_{k}=\frac{\sum_{\mathbf{x}}\left|\operatorname{det}\left(e^{i k_{a} x_{b}}\right)_{a, b}\right|^{4}}{L^{2 M}}
$$

Next, set $\theta_{j}:=\frac{2 \pi}{L} x_{j}$, so that

$$
e^{i k_{a} x_{b}}=e^{i I_{a} \theta_{b}}
$$

We set

$$
c(\boldsymbol{\theta})=\operatorname{det}\left(e^{i I_{a} \theta_{b}}\right)_{a, b}
$$

and introduce the notation $\sum_{\boldsymbol{\theta}}$ to denote summation over distinct values of $\theta_{1}, \ldots, \theta_{M}$ in $\frac{2 \pi}{L}\{0, \ldots, L-1\}$.

Example 1. IPR of the ground state. For the ground state,

$$
\left\{I_{1}, I_{2}, \ldots, I_{M}\right\}=\left\{\frac{M-1}{2}, \frac{M-1}{2}-1, \ldots,-\frac{M-1}{2}\right\}
$$

Therefore

$$
c(\boldsymbol{\theta})=\operatorname{det}\left(\begin{array}{cccc}
e^{i\left(-\frac{M-1}{2}\right) \theta_{1}} & e^{i\left(-\frac{M-1}{2}\right) \theta_{2}} & \cdots & e^{i\left(-\frac{M-1}{2}\right) \theta_{M}} \\
e^{i\left(-\frac{M-1}{2}+1\right) \theta_{1}} & e^{i\left(-\frac{M-1}{2}+1\right) \theta_{2}} & \cdots & e^{i\left(-\frac{M-1}{2}+1\right) \theta_{M}} \\
\vdots & \vdots & \cdots & \vdots \\
e^{i\left(\frac{M-1}{2}\right) \theta_{1}} & e^{i\left(\frac{M-1}{2}\right) \theta_{2}} & \cdots & e^{i\left(\frac{M-1}{2}\right) \theta_{M}}
\end{array}\right) .
$$

We notice that (26) is equal to

$$
e^{i\left(-\frac{M-1}{2}\right)\left(\theta_{1}+\ldots+\theta_{M}\right)} \operatorname{det}\left(\begin{array}{cccc}
1 & 1 & \cdots & 1 \\
e^{i \theta_{1}} & e^{i \theta_{2}} & \cdots & e^{i \theta_{M}} \\
\vdots & \vdots & \cdots & \vdots \\
e^{i(M-1) \theta_{1}} & e^{i(M-1) \theta_{2}} & \cdots & e^{i(M-1) \theta_{M}}
\end{array}\right),
$$


the determinant being a Vandermonde determinant:

$$
\operatorname{det}\left(\begin{array}{cccc}
1 & 1 & \cdots & 1 \\
e^{i \theta_{1}} & e^{i \theta_{2}} & \cdots & e^{i \theta_{M}} \\
\vdots & \vdots & \cdots & \vdots \\
e^{i(M-1) \theta_{1}} & e^{i(M-1) \theta_{2}} & \cdots & e^{i(M-1) \theta_{M}}
\end{array}\right)=\prod_{j<k}\left(e^{i \theta_{k}}-e^{i \theta_{j}}\right) .
$$

Therefore the IPR of the ground state is equal to

$$
\mathbf{t}_{0}=\frac{1}{L^{2 M}} \sum_{\boldsymbol{\theta}} \prod_{j<k}\left|e^{i \theta_{k}}-e^{i \theta_{j}}\right|^{4} .
$$

We will complete the evaluation in (44).

Notice that $\left|\operatorname{det}\left(e^{i I_{a} \theta_{b}}\right)_{a, b}\right|$ is invariant under shifts of the Bethe roots $I$, since

$$
\operatorname{det}\left(e^{i\left(I_{a}+n\right) \theta_{b}}\right)_{a, b}=e^{i n\left(\theta_{1}+\ldots+\theta_{M}\right)} \operatorname{det}\left(e^{i I_{a} \theta_{b}}\right)_{a, b} .
$$

Therefore IPRs corresponding to two excited states whose Bethe roots are related by a shift will be equal.

Assume without loss of generality that $I_{1}>I_{2}>\ldots>I_{M}$. Set

$$
\lambda_{j}=I_{j}-\frac{M+1}{2}+j
$$

so that $\lambda_{1} \geq \lambda_{2} \geq \ldots \geq \lambda_{M}$ yields a partition $\lambda=\left[\lambda_{1}, \ldots, \lambda_{M}\right]$. In this notation,

$$
\left|\operatorname{det}\left(e^{i I_{a} \theta_{b}}\right)_{a, b}\right|=\left|s_{\lambda}\left(e^{i \theta_{1}}, \ldots, e^{i \theta_{M}}\right) V\left(e^{i \theta_{1}}, \ldots, e^{i \theta_{M}}\right)\right|,
$$

where $s$ is the Schur polynomial (discussed in Section 5) and $V\left(x_{1}, \ldots, x_{M}\right)$ is the Vandermonde determinant:

$$
V\left(x_{1}, \ldots, x_{M}\right)=\prod_{i<j}\left(x_{j}-x_{i}\right) .
$$

We then have

$$
\mathbf{t}_{\lambda}=\frac{\sum_{\boldsymbol{\theta}}\left|s_{\lambda}\left(e^{i \theta_{1}}, \ldots, e^{i \theta_{M}}\right)\right|^{4}\left|\prod_{i<j}\right| e^{i \theta_{i}}-\left.e^{i \theta_{j}}\right|^{4}}{L^{2 M}},
$$

where we have introduced a labeling by partitions $\lambda$.

\section{Connection Between the IPRs of XX, the Circular symplectic ensemble and the Dyson-Gaudin Coulomb gas}

We show in this section how to interpret the IPRs of XX in terms of expectation values of quantities for two-dimensional Coulomb gas on a one-dimensional lattice, or equivalently, as expectation values over a discretization of the circular symplectic ensemble.

In studying his famous random matrix ensembles, Dyson Dys62 developed a physical model for the eigenvalues of the matrices of the circular ensemble consisting of charges distributed on a unit circle in two-dimensions and experiencing Coulomb forces. In Gau73, Gaudin studied a discrete formulation of the problem, in which the gas particles lie on lattice sites of the circle. This formulation corresponds to the finite $L$ situation of the IPRs above, whereas Dyson's to the limit as $L \rightarrow \infty$. We will now explain the details. 
4.1. Circular symplectic ensemble. The circular symplectic ensemble (CSE) is the space of self dual unitary quaternion matrices with a probability measure defined as follows. Each element is a unitary matrix, so it has eigenvalues on the unit circle $e^{i \theta_{1}}, \ldots, e^{i \theta_{M}}$. The probability density function for the phases in the circular symplectic ensemble is given by

$$
p\left(\theta_{1}, \ldots, \theta_{M}\right)=\frac{1}{Z_{M, 4}} \prod_{1 \leq i<j \leq M}\left|e^{i \theta_{i}}-e^{i \theta_{j}}\right|^{4} .
$$

The normalization constant is given by

$$
Z_{M, 4}=(2 \pi)^{M} \frac{(2 M) !}{2^{M}} .
$$

4.2. Dyson-Gaudin Coulomb gas. The positions which a unit charge can occupy on the circumference of a unit circle are restricted to $L$ equidistant points $\exp \left(i \theta_{j}\right), \theta_{j}=2 \pi j / L, 1 \leq j \leq L$. One considers three distinguished values of the inverse temperature $\beta=1,2$, 便 The joint probability density for $M$ unit charges to occupy positions $j_{1}, \ldots, j_{M}$ is given by

$$
P_{\beta}\left(j_{1}, \ldots, j_{M}\right)=C_{L M \beta}^{-1} L^{-M} \exp (-\beta W) .
$$

Here $W$ is the potential energy, calculated as follows. If we place point unit charges at angles $\theta_{1}, \ldots, \theta_{M}$ on a circle in two dimensions of radius 1 , then the potential energy is equal to

$$
W=-\sum_{1 \leq j<k \leq M} \log \left|e^{i \theta_{k}}-e^{i \theta_{j}}\right|, \quad \theta_{l}=2 \pi j_{l} / L .
$$

Note in particular that

$$
\exp (-\beta W)=\prod_{1 \leq j<k \leq M}\left|e^{i \theta_{k}}-e^{j \theta_{j}}\right|^{\beta} .
$$

The expected value of a quantity $f\left(e^{i \theta_{1}}, \ldots, e^{i \theta_{M}}\right)$ is given by

$$
\mathbb{E}_{\beta}(f)=\sum_{\boldsymbol{\theta}} f\left(e^{i \theta_{1}}, \ldots, e^{i \theta_{M}}\right) P_{\beta}\left(j_{1}, \ldots, j_{M}\right)=C_{L M \beta}^{-1} L^{-M} \sum_{\boldsymbol{\theta}} f\left(e^{i \theta_{1}}, \ldots, e^{i \theta_{M}}\right) \prod_{1 \leq j<k \leq M}\left|e^{i \theta_{k}}-e^{i \theta_{j}}\right|^{\beta} .
$$

In Gau73, Gaudin calculates the partition function of the discrete Coulomb gas. In particular, he determines the normalization constant for $\beta=4$ :

$$
\sum_{\boldsymbol{\theta}} \prod_{1 \leq j<k \leq M}\left|e^{i \theta_{k}}-e^{i \theta_{j}}\right|^{4}=\frac{(2 M) ! L^{M}}{2^{M} M !}
$$

Hence

$$
\mathbb{E}_{\beta=4}(f)=\frac{2^{M} M !}{(2 M) ! L^{M}} \sum_{\boldsymbol{\theta}} f\left(e^{i \theta_{1}}, \ldots, e^{i \theta_{M}}\right) \prod_{1 \leq j<k \leq M}\left|e^{i \theta_{k}}-e^{i \theta_{j}}\right|^{4} .
$$

We recognize that,

$$
\mathbf{t}_{\lambda}=\frac{(2 M) !}{2^{M} M ! L^{M}} \mathbb{E}_{\beta=4}\left(\left|s_{P}\right|^{4}\right) .
$$

Thus the IPR of the ground state is equal to

$$
\mathbf{t}_{0}=\frac{(2 M) !}{2^{M} M ! L^{M}}
$$

as determined previously in MPL16] for $L=2 M$.

\footnotetext{
${ }^{1}$ These correspond respectively to the orthogonal, unitary and symplectic circular ensembles, which are closely related to Dyson's threefold way.
} 


\section{JACK AND SCHUR POLYNOMIALS}

In this section we gather the results from symmetric function theory that we will use to analyze the IPRs. The primary sources are [Sta99] and Mac15].

5.1. Partitions. A partition $\lambda=\left[\lambda_{1}, \lambda_{2}, \ldots\right]$ is a weakly decreasing sequence of nonnegative integers. The length $l(\lambda)$ is the number of nonzero entries. Let $m_{i}(\lambda)=m_{i}$ be the number of times $i$ appears in $\lambda$. Set

$$
z_{\lambda}=\prod_{i \geq 1} i^{m_{i}} m_{i} !
$$

The weight of a partition $|\lambda|$ is $\sum_{i} \lambda_{i}$. Given two partitions $\lambda, \mu$ of equal weight, the dominance partial order is defined by

$$
\lambda \succeq \mu \Longleftrightarrow \sum_{i=1}^{k} \lambda_{i} \geq \sum_{i=1}^{k} \mu_{i} \forall k .
$$

The Young diagram of a partition $\lambda$ is a convenient graphical representation. It is obtained by putting $\lambda_{i}$ boxes left-aligned at row $i$. The conjugate partition $\lambda^{\prime}$ of $\lambda$ is obtained by reflecting the Young diagram of $\lambda$ about the diagonal.

Example 2. Young diagrams and conjugate partitions. The Young diagram of $\lambda=[2,1,1]$ is

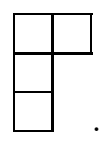

The conjugate partition $\lambda^{\prime}$ is read off from the Young diagram after reflection in the diagonal:

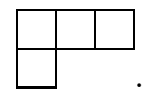

Thus $\lambda^{\prime}=[3,1]$.

5.2. Jack polynomials. The Jack polynomials $J_{\lambda}^{(2 / \beta)}$ are a notable family of symmetric polynomials parameterized by a real parameter $\beta$. For $\beta=2$, they specialize to scaled Schur functions. For $\beta=1$ one obtains the zonal polynomials and for $\beta=4$ the quaternion zonal polynomials [DES07. We will be using the "J" normalization. The Jack polynomials will be useful for us due to their property of orthogonalizing the circular ensembles [Mac15, (10.36)]. We state this more precisely now.

Let $\lambda$ and $\kappa$ be a pair of partitions. Let $\lambda^{\prime}$ denote the transpose of the partition $\lambda$ and $l(\lambda)$ the number of nonzero parts in $\lambda$. Let $(i, j) \in \lambda$ refer to a cell of the Young diagram of $\lambda$ (the indexing begins at $(1,1))$. Set

$$
\mathcal{N}_{\lambda}^{(\alpha)}(M)=\prod_{(i, j) \in \lambda} \frac{M+(j-1) \alpha-(i-1)}{M+j \alpha-i}
$$

and

$$
C_{\lambda}(\alpha)=\prod_{(i, j) \in \lambda}\left(\alpha\left(\lambda_{i}-j\right)+\lambda_{j}^{\prime}-i+1\right)\left(\alpha\left(\lambda_{i}-j\right)+\lambda_{j}^{\prime}-i+\alpha\right) .
$$

The Jack polynomials satisfy JM15.

$$
\begin{gathered}
\int_{[0,2 \pi]^{M}} \frac{d \theta_{1}}{2 \pi} \ldots \frac{d \theta_{M}}{2 \pi} J_{\kappa}^{(2 / \beta)}\left(e^{i \theta_{1}}, \ldots, e^{i \theta_{n}}\right) \overline{J_{\lambda}^{(2 / \beta)}\left(e^{i \theta_{1}}, \ldots, e^{i \theta_{n}}\right)} \prod_{j<k}\left|e^{i \theta_{j}}-e^{i \theta_{k}}\right|^{\beta} \\
=\delta_{\kappa, \lambda} \delta(l(\lambda) \leq M) C_{\lambda}(2 / \beta) \mathcal{N}_{\lambda}^{(2 / \beta)}(M) \frac{\Gamma(1+M \beta / 2)}{\Gamma(1+\beta / 2)^{M}} . \\
7
\end{gathered}
$$


The factor $\frac{\Gamma(1+M \beta / 2)}{\Gamma(1+\beta / 2)^{M}}$ is the one from Dyson's ex-conjecture Dys62,

$$
\int_{[0,2 \pi]^{M}} \frac{d \theta_{1}}{2 \pi} \cdots \frac{d \theta_{M}}{2 \pi} \prod_{j<k}\left|e^{i \theta_{j}}-e^{i \theta_{k}}\right|^{\beta}=\frac{\Gamma(1+M \beta / 2)}{\Gamma(1+\beta / 2)^{M}} .
$$

The factor $\delta(l(\lambda) \leq M)$ is simply the statement that $J_{\lambda}^{(2 / \beta)}=0$ if the number of parts of $\lambda$ is greater than $M$.

We define a corresponding inner product

$$
\langle f, g\rangle_{\beta}=\frac{1}{M !} \int_{[0,2 \pi]^{M}} \frac{d \theta_{1} \cdots d \theta_{M}}{(2 \pi)^{M}} f \bar{g} \prod_{j<k}\left|e^{i \theta_{j}}-e^{i \theta_{k}}\right|^{\beta}
$$

with respect to which

$$
\left\langle J_{\kappa}^{(2 / \beta)}, J_{\lambda}^{(2 / \beta)}\right\rangle_{\beta}=\delta_{\kappa, \lambda} C_{\lambda}(2 / \beta) \mathcal{N}_{\lambda}^{(2 / \beta)}(M) \frac{\Gamma(1+M \beta / 2)}{M ! \Gamma(1+\beta / 2)^{M}} .
$$

This inner product can be thought of as a specialization of the Hall inner product to finitely many variables. It may also be written as the extraction of a constant term (see e.g., [Mac15, VI, 10.35])

$$
\begin{gathered}
\langle f, g\rangle_{\beta}=\frac{1}{M !} \int_{T^{M}} f(z) \overline{g(z)} \Delta(z ; \beta)=\frac{1}{M !} \operatorname{CT}[f \bar{g} \Delta(z ; \beta)] \\
\Delta(z ; \beta)=\prod_{i \neq j}\left(1-z_{i} z_{j}^{-1}\right)^{\beta / 2}, \quad T=\{z:|z|=1\} .
\end{gathered}
$$

A word about notation. Since our main interest will be with $\beta=4$, we will reserve the shorter $\mathcal{N}_{\lambda}, \mathcal{C}_{\lambda}$ for that case.

The Jack polynomials also form an orthogonal basis of the symmetric polynomials with respect to the Hall inner product [JM15, (4.1),(4.3)],

$$
\left\langle J_{\lambda}^{(2 / \beta)}, J_{\mu}^{(2 / \beta)}\right\rangle_{\beta}^{\prime}=\delta_{\lambda \mu} C_{\lambda}(2 / \beta)
$$

defined on power sum polynomials by

$$
\left\langle p_{\lambda}, p_{\mu}\right\rangle_{\beta}^{\prime}=\delta_{\lambda \mu}(2 / \beta)^{l(\lambda)} z_{\lambda} .
$$

Conceptually, we will think of this inner product as being obtained from $\langle\cdot, \cdot\rangle_{\beta}$, upon approximating $\mathcal{N}_{\lambda}^{(\beta)}(M) \approx 1$, which occurs for large $M$. The $\Gamma$ factors are a matter of normalization.

5.3. Schur polynomials. Let $s_{\lambda}\left(x_{1}, \ldots, x_{M}\right)$ denote the Schur polynomial associated to the partition $\lambda=\left(\lambda_{1}, \ldots, \lambda_{M}\right)$ :

$$
s_{\lambda}\left(x_{1}, \ldots, x_{M}\right):=\frac{\operatorname{det}\left[\left(x_{i}^{\lambda_{j}+M-j}\right)_{1 \leq i, j \leq M}\right]}{\operatorname{det}\left[\left(x_{i}^{M-j}\right)_{1 \leq i, j \leq M}\right]} .
$$

The Schur polynomials are (rescaled) Jack polynomials $J^{(1)}$ and form a linear basis for the symmetric polynomials. There is a combinatorial description for the product of two Schur functions. Namely, writing

$$
s_{\lambda} s_{\mu}=\sum_{\nu} c_{\lambda, \mu}^{\nu} s_{\nu}
$$

the Littlewood-Richardson rule states that $c_{\lambda, \mu}^{\nu}$ is equal to the number of Littlewood-Richardson tableaux of skew shape $\nu / \lambda$ and of weight $\mu$. The coefficients are known as the Littlewood-Richardson coefficients, and appear in many other mathematical contexts. 
5.4. Transition matrices. When discussing transition matrices between $\mathbb{Q}$-bases of symmetric polynomials [Mac15, I §6], we index rows and columns by partitions of a positive integer $n$, arranged in reverse lexicographical order (so that $[n]$ is first and $\left[1^{n}\right]$ is last). A matrix $\left(M_{\lambda \mu}\right)$ is strictly upper triangular if $M_{\lambda \mu}=0$ unless $\mu \preceq \lambda$ in the dominance order on partitions. The strictly upper triangular matrices form a group. Given two $\mathbb{Q}$-bases $\left(u_{\lambda}\right),\left(v_{\lambda}\right)$, we denote by $M(u, v)$ the matrix $\left(M_{\lambda \mu}\right)$ of coefficients in the equations

$$
u_{\lambda}=\sum_{\mu} M_{\lambda \mu} v_{\mu}
$$

$M(u, v)$ is called the transition matrix from the basis $\left(u_{\lambda}\right)$ to the basis $\left(v_{\lambda}\right)$.

The following result makes appearance in experimental form in Gom14 and is derived using physical arguments in [WY11.

Proposition 1. The transition matrix $M\left(s, J^{(2 / \beta)}\right)$ is strictly upper triangular with respect to the dominance ordering of partitions.

Proof. Let $\left(m_{\lambda}\right)$ denote the the monomial basis for the symmetric polynomials. The transition matrices $M(s, m)$ and $M\left(J^{(2 / \beta)}, m\right)$ are strictly upper triangular. Consequently,

$$
M\left(s, J^{(2 / \beta)}\right)=M(s, m) M\left(m, J^{(2 / \beta)}\right)=M(s, m) M\left(J^{(2 / \beta)}, m\right)^{-1}
$$

is strictly upper triangular.

Define the transition coefficients $d_{\nu}^{\lambda}(\beta)=d_{\nu}$ from Schur polynomials to Jack polynomials:

$$
s_{\lambda}\left(x_{1}, \ldots, x_{n}\right)=\sum_{\nu \preceq \lambda} d_{\nu}^{\lambda} J_{\nu}^{(2 / \beta)}\left(x_{1}, \ldots, x_{n}\right) .
$$

\section{Calculating the IPRs of XX}

Set

$$
\Delta(x ; \beta)=\prod_{i \neq j}\left(1-x_{i} x_{j}^{-1}\right)^{\beta / 2} .
$$

Then

$$
\Delta(x ; \beta)=\prod_{i<j}\left[\left(x_{j}-x_{i}\right)\left(x_{j}^{-1}-x_{i}^{-1}\right)\right]^{\beta / 2} .
$$

In particular,

$$
\Delta\left(e^{i \theta} ; \beta\right)=\prod_{k<l}\left|e^{i \theta_{l}}-e^{i \theta_{k}}\right|^{\beta} .
$$

Define $T_{L}$ to be a discrete torus:

$$
T_{L}:=\left\{e^{i \theta} \in \mathbb{C}: \theta \in \frac{2 \pi \mathbb{Z}}{L} \forall j\right\} .
$$

Define a scalar product on symmetric polynomials by

$$
\langle f, g\rangle_{L ; \beta}:=\frac{1}{L^{M} M !} \sum_{T_{L}^{M}} f(z) \bar{g}(z) \Delta(z ; \beta) .
$$

In terms of this "discrete" Hall inner product,

$$
\mathbf{t}_{\lambda}=\frac{\left\langle s_{\lambda}^{2}, s_{\lambda}^{2}\right\rangle_{L ; 4}}{L^{M}} .
$$


Note that

$$
\sum_{z_{1}, \ldots, z_{M} \in T_{L}} z_{1}^{a_{1}} z_{2}^{a_{2}} \cdots z_{M}^{a_{M}}= \begin{cases}L^{M} & \text { if } a_{1} \equiv a_{2} \equiv \cdots \equiv a_{M} \equiv 0 \quad(\bmod L) \\ 0 & \text { otherwise. }\end{cases}
$$

Consequently, the inner product can be expressed as an extraction of coefficients:

$$
\langle f, g\rangle_{L ; \beta}=\frac{1}{M !} \sum_{i_{1}, \ldots, i_{M} \in \mathbb{Z}}\left[x_{1}^{L i_{1}} \cdots x_{M}^{L i_{M}}\right] f\left(x_{1}, \ldots, x_{M}\right) g\left(x_{1}^{-1}, \ldots, x_{M}^{-1}\right) \Delta(x ; \beta) .
$$

As we show next, for fixed $f, g, M$ and all sufficiently large $L$,

$$
\langle f, g\rangle_{L ; \beta}=\frac{1}{M !} \operatorname{CT}\left[f(x) g\left(x^{-1}\right) \Delta(x ; \beta)\right]=\frac{1}{M !} \int_{T} f(z) \overline{g(z)} \Delta(z ; \beta),
$$

the Hall inner product of (53).

Proposition 2. Let $f, g$ be symmetric polynomials and suppose that $\beta / 2 \in \mathbb{N}$. Let $\operatorname{deg}_{i}(f)$ denote the degree of $x_{i}$ in $f$. Set

$$
\begin{aligned}
p_{1} & :=\max _{i} \operatorname{deg}_{i}(f), \quad q_{1}:=\max _{i} \operatorname{deg}_{i}(g) . \\
p_{2} & :=\min _{i} \operatorname{deg}_{i}(f), \quad q_{2}:=\min _{i} \operatorname{deg}_{i}(g) .
\end{aligned}
$$

Then

$$
\left|\max _{i} \operatorname{deg}_{i}(f \bar{g} \Delta(x ; \beta))\right| \leq \max \left\{\left|p_{1}-q_{2}\right|,\left|p_{2}-q_{1}\right|\right\}+(\beta / 2)(M-1) .
$$

Proof. Let $\delta=(0,1, \ldots, M-1)$. The product $f \bar{g} \Delta(x ; \beta)$ is a sum of monomials of the following form: with $\sigma^{\left(i_{1}\right)}, \sigma^{\left(i_{2}\right)}$ permutations, $x_{1}^{f_{1}} \cdots x_{M}^{f_{M}}$ monomials coming from $f$ and $x_{1}^{g_{1}} \cdots x_{M}^{g_{M}}$ monomials from $g$,

$$
x_{1}^{f_{1}} \cdots x_{M}^{f_{M}} x_{1}^{-g_{1}} \cdots x_{M}^{-g_{M}} \prod_{i=1}^{\beta / 2}\left(x^{\sigma^{\left(i_{1}\right)} \delta} x^{-\sigma^{\left(i_{2}\right)} \delta}\right) .
$$

The degree of $x_{i}$ is equal to

$$
f_{i}-g_{i}+\sum_{i=1}^{\beta / 2} \sigma^{\left(i_{1}\right)}(\delta)-\sigma^{\left(i_{2}\right)}(\delta)
$$

It satisfies

$$
\left|f_{i}-g_{i}+\sum_{i=1}^{\beta / 2} \sigma^{\left(i_{1}\right)}(\delta)-\sigma^{\left(i_{2}\right)}(\delta)\right| \leq \max \left\{\left|p_{1}-q_{2}\right|,\left|p_{2}-q_{1}\right|\right\}+(\beta / 2)(M-1) .
$$

Example 3. Proposition 2 is tight. Taking $M=4, \lambda=[2,1,1]$ and $\beta=2$, we have

$$
p_{1}=q_{1}=2, \quad p_{2}=q_{2}=0 .
$$

The proposition guarantees that the maximum degree of a variable in $f \bar{g} \Delta(x ; 2)$ does not exceed $M+1=$ 5. This means that when $L>5$, the proposition guarantees that the discrete Hall inner product will be equal to the Hall inner product. Computing on the monomial symmetric functions,

$$
\begin{aligned}
\left\langle m_{\lambda}, m_{\lambda}\right\rangle_{4 ; 2} & =16 \\
\left\langle m_{\lambda}, m_{\lambda}\right\rangle_{5 ; 2} & =9 \\
\left\langle m_{\lambda}, m_{\lambda}\right\rangle_{L ; 2}=\frac{1}{M !} \mathrm{CT}\left[m_{\lambda} \bar{m}_{\mu} \Delta(x)\right] & =\left\langle m_{\lambda}, m_{\lambda}\right\rangle_{2}=2 \quad \forall L \geq 6 .
\end{aligned}
$$

We see that the proposition is tight in the sense that no smaller $L$ would work. 
Proposition 2 clarifies when we can truncate the sum in (69). Namely, each $i$ should satisfy

$$
L i \leq \max \left\{\left|p_{1}-q_{2}\right|,\left|p_{2}-q_{1}\right|\right\}+(\beta / 2)(M-1) .
$$

The case $\beta=4$ is of particular interest to us. According to Proposition 2 , if $M, L, \lambda, \mu$ satisfy $L-2 M>\max \left\{\lambda_{1}, \mu_{1}\right\}-2$, then

$$
\left\langle J_{\lambda}^{(1 / 2)}, J_{\mu}^{(1 / 2)}\right\rangle_{L ; 4}=\left\langle J_{\lambda}^{(1 / 2)}, J_{\mu}^{(1 / 2)}\right\rangle_{4}
$$

In particular, under these conditions these Jack polynomials are orthogonal with respect to the scalar product $\langle\cdot, \cdot\rangle_{L ; 4}$.

Theorem 1. Suppose that $L$ and $M$ are given. Let $\lambda$ be a fixed partition with $\lambda_{1}<(1 / 2)(L-2 M+2)$. Let $s_{\lambda}, J_{\mu}^{(1 / 2)}$ be the Schur and Jack polynomials, respectively. Write

$$
s_{\lambda}^{2}=\sum_{\nu} r_{\lambda}^{\nu} J_{\nu}^{(1 / 2)}
$$

The IPR for partition $\lambda$ is equal to

$$
\mathbf{t}_{\lambda}=\frac{(2 M) !}{M !(2 L)^{M}} \sum_{\nu} \mathcal{N}_{\nu}^{(1 / 2)}(M) \mathcal{C}_{\nu}(1 / 2)\left(r_{\lambda}^{\nu}\right)^{2} .
$$

Proof. Under these conditions on $\lambda$,

$$
\mathbf{t}_{\lambda}=\frac{\left\langle s_{\lambda}^{2}, s_{\lambda}^{2}\right\rangle_{L ; 4}}{L^{M}}=\frac{\left\langle s_{\lambda}^{2}, s_{\lambda}^{2}\right\rangle_{4}}{L^{M}}
$$

We have

$$
\left\langle s_{\lambda}^{2}, s_{\lambda}^{2}\right\rangle_{4}=\left\langle\sum_{\nu} r_{\lambda}^{\nu} J_{\nu}^{(1 / 2)}, \sum_{\nu} r_{\lambda}^{\nu} J_{\nu}^{(1 / 2)}\right\rangle_{4}=\sum_{\nu}\left(r_{\lambda}^{\nu}\right)^{2}\left\langle J_{\nu}^{(1 / 2)}, J_{\nu}^{(1 / 2)}\right\rangle_{4}
$$

Applying (52) for $\beta=4$ yields the result.

Example 4. The simplest excited state has $\lambda=[1]$. Computing,

$$
s_{[1]}^{2}=s_{[1,1]}+s_{[2]} \text {. }
$$

and

$$
s_{[1,1]}=(1 / 2) J_{[1,1]}, \quad s_{[2]}=(2 / 3) J_{[2]}-(1 / 6) J_{[1,1]},
$$

so that

$$
s_{[1]}^{2}=(2 / 3) J_{[2]}+(1 / 3) J_{[1,1]}
$$

The IPR is

$$
\mathbf{t}_{[1]}=\left(1 / L^{M}\right)\left\langle s_{[1]}^{2}, s_{[1]}^{2}\right\rangle_{L}=\left(1 / L^{M}\right)\left\langle(2 / 3) J_{[2]}+(1 / 3) J_{[1,1]},(2 / 3) J_{[2]}+(1 / 3) J_{[1,1]}\right\rangle_{L} .
$$

We also compute

$$
\mathcal{N}_{[1,1]}=\frac{M}{M-1 / 2} \frac{M-1}{M-3 / 2}, \quad \mathcal{N}_{[2]}=\frac{M}{M-1 / 2} \frac{M+1 / 2}{M}
$$

Consequently,

$$
L^{M} \mathbf{t}_{[1]}=(4 / 9)\left\langle J_{[2]}, J_{[2]}\right\rangle_{L}+(4 / 9)\left\langle J_{[2]}, J_{[1,1]}\right\rangle_{L}+(1 / 9)\left\langle J_{[1,1]}, J_{[1,1]}\right\rangle_{L} .
$$

Assuming $L>2 M+2$, we can replace the discrete Hall inner products with the Hall inner product. Then

$$
\left\langle J_{[2]}, J_{[2]}\right\rangle_{L}=C_{[2]} \mathcal{N}_{[2]} \frac{(2 M) !}{M ! 2^{M}}, \quad\left\langle J_{[1,1]}, J_{[1,1]}\right\rangle_{L}=C_{[1,1]} \mathcal{N}_{[1,1]} \frac{(2 M) !}{M ! 2^{M}}, \quad\left\langle J_{[1,1]}, J_{[2]}\right\rangle_{L}=0 .
$$


This gives us the exact value of the IPR $\mathbf{t}_{[1]}$. Assuming further that $M$ is large, so that $\mathcal{N} \approx 1$, yields

$$
\mathbf{t}_{[1]} \approx(1 / 2) \frac{(2 M) !}{M ! L^{M} 2^{M}}=(1 / 2) \mathbf{t}_{0} .
$$

Example 5. Table of IPRs.

\begin{tabular}{|l|l|}
\hline$\lambda$ & $\mathbf{t}_{\lambda}$ \\
\hline[] & $\mathbf{t}_{0}$ \\
\hline$[1]$ & $(1 / 2) \mathbf{t}_{0}$ \\
\hline$[2]$ & $(11 / 32) \mathbf{t}_{0}$ \\
\hline$[1,1]$ & $(11 / 32) \mathbf{t}_{0}$ \\
\hline$[3]$ & $(17 / 64) \mathbf{t}_{0}$ \\
\hline$[2,1]$ & $(1 / 4) \mathbf{t}_{0}$ \\
\hline$[1,1,1]$ & $(17 / 64) \mathbf{t}_{0}$ \\
\hline$[4]$ & $(1787 / 8192) \mathbf{t}_{0}$ \\
\hline$[3,1]$ & $(1451 / 8192) \mathbf{t}_{0}$ \\
\hline$[2,2]$ & $(99 / 512) \mathbf{t}_{0}$ \\
\hline$[2,1,1]$ & $(1451 / 8192) \mathbf{t}_{0}$ \\
\hline$[1,1,1,1]$ & $(1787 / 8192) \mathbf{t}_{0}$ \\
\hline
\end{tabular}

TABLE 1. Table of IPRs, assuming $L-2 M>2 \lambda_{1}$ and $\mathcal{N} \approx 1$, the latter occurring when $M$ is large.

The table suggests the following property:

$$
\left\langle J_{\lambda}^{(1)}, J_{\lambda}^{(1)}\right\rangle_{4}^{\prime}=\left\langle J_{\lambda^{\prime}}^{(1)}, J_{\lambda^{\prime}}^{(1)}\right\rangle_{4}^{\prime} .
$$

Here, we recall, $J^{(1)}$ is a (rescaled) Schur polynomial and the inner product is the Hall inner product for parameter $\beta=4$ (or $\alpha=1 / 2$ in the symmetric polynomial literature). We emphasize that the inner product has a different parameter than the Jack polynomials being operated on. Moreover, generally even

$$
\left\langle J_{\lambda}^{(1 / 2)}, J_{\lambda}^{(1 / 2)}\right\rangle_{4}^{\prime} \neq\left\langle J_{\lambda^{\prime}}^{(1 / 2)}, J_{\lambda^{\prime}}^{(1 / 2)}\right\rangle_{4}^{\prime},
$$

where the parameters are matching. Thus the choice of Schur polynomials is special among the Jack polynomials.

Theorem 2. Let $\lambda, \mu$ be partitions and $s_{\lambda}$ the Schur polynomial. For any $\beta$,

$$
\left\langle s_{\lambda}, s_{\mu}\right\rangle_{\beta}^{\prime}=\left\langle s_{\lambda^{\prime}}, s_{\mu^{\prime}}\right\rangle_{\beta}^{\prime}
$$

Proof. Following [Mac15, VI §2], consider the symmetric polynomials with coefficients in $\mathbb{Q}(q, t)$ and define an inner product on the basis of power sum polynomials $p_{\mu}$ :

$$
z_{\mu}(q, t):=z_{\mu} \prod_{i=1}^{l(\mu)} \frac{1-q^{\mu_{i}}}{1-t^{\mu_{i}}}, \quad\left\langle p_{\mu}, p_{\kappa}\right\rangle_{q, t}^{\prime}:=\delta_{\mu, \kappa} z_{\mu}(q, t) .
$$

This inner product is a q-analogue of the inner product $\langle\cdot, \cdot\rangle_{\beta}^{\prime}$. Indeed, denote the limit $(q, t) \rightarrow(1,1)$ with $q=t^{2 / \beta}$ by $(q, t) \underset{2 / \beta}{\longrightarrow}(1,1)$. Then

$$
\lim _{(q, t) \underset{2 / \beta}{\longrightarrow}(1,1)}\langle\cdot, \cdot\rangle_{(q, t)}^{\prime}=\langle\cdot, \cdot\rangle_{\beta}^{\prime} .
$$

Let $\omega_{t, q}$ be the standard automorphism on symmetric functions with coefficients in $\mathbb{Q}(q, t)$ :

$$
\omega_{q, t}\left(p_{\lambda}\right)=(-1)^{|\lambda|+l(\lambda)} p_{\lambda} \prod_{i=1}^{l(\lambda)} \frac{1-q^{\lambda_{i}}}{1-t^{\lambda_{i}}}
$$


Let

$$
\omega_{\beta}=\lim _{(q, t) \underset{2 / \beta}{\longrightarrow}(1,1)} \omega_{q, t} .
$$

The automorphism $\omega_{q, t}$ satisfies

$$
\begin{gathered}
\omega_{q, t}^{-1}=\omega_{t, q} \\
\left\langle\omega_{u, v} f, g\right\rangle_{q, t}^{\prime}=\left\langle f, \omega_{u, v} g\right\rangle_{q, t}^{\prime} \\
\left\langle\omega_{t, q} f, g\right\rangle_{q, t}^{\prime}=\left\langle\omega_{2} f, g\right\rangle_{2}^{\prime} \\
\left\langle\omega_{2} f, \omega_{2} g\right\rangle_{2}^{\prime}=\langle f, g\rangle_{2}^{\prime} .
\end{gathered}
$$

The Schur polynomials satisfy

$$
\omega_{2} s_{\lambda}=s_{\lambda^{\prime}}
$$

Putting this together,

$$
\left\langle s_{\lambda}, s_{\mu}\right\rangle_{\beta}^{\prime}=\left\langle\omega_{\beta}^{-1} s_{\lambda}, \omega_{\beta} s_{\mu}\right\rangle_{\beta}^{\prime}=\left\langle\omega_{2} s_{\lambda}, \omega_{\beta} s_{\mu}\right\rangle_{2}^{\prime} .
$$

By evaluating on the power sum basis, we can check that

$$
\omega_{q, t} \omega_{2}=\omega_{2} \omega_{q, t} .
$$

Consequently,

$$
\left\langle\omega_{2} s_{\lambda}, \omega_{\beta} s_{\mu}\right\rangle_{2}^{\prime}=\left\langle\omega_{2} \omega_{2} s_{\lambda}, \omega_{2} \omega_{\beta} s_{\mu}\right\rangle_{2}^{\prime}=\left\langle\omega_{2} \omega_{2} s_{\lambda}, \omega_{\beta} \omega_{2} s_{\mu}\right\rangle_{2}^{\prime}=\left\langle\omega_{2} s_{\lambda^{\prime}}, \omega_{\beta} s_{\mu^{\prime}}\right\rangle_{2}^{\prime}
$$

which proves the result.

Corollary 1. Let $\lambda$ be a partition satisfying $\lambda_{1}<(1 / 2)(L-2 M+2)$ with $M \gg|\lambda|\left(\right.$ so that $\left.\mathcal{N}_{\lambda} \approx 1\right)$. Then the IPRs satisfy the duality relation

$$
\mathbf{t}_{\lambda}=\mathbf{t}_{\lambda^{\prime}}
$$

Proof. We have shown that under these hypotheses,

$$
\mathbf{t}_{\lambda}=\frac{\left\langle s_{\lambda}^{2}, s_{\lambda}^{2}\right\rangle_{4}}{L^{M}}
$$

Moreover, since $\mathcal{N}_{\lambda} \approx 1$

$$
\frac{\left\langle s_{\lambda}^{2}, s_{\lambda}^{2}\right\rangle_{4}}{\left\langle s_{\lambda^{\prime}}^{2}, s_{\lambda^{\prime}}^{2}\right\rangle_{4}}=\frac{\left\langle s_{\lambda^{\prime}}^{2}, s_{\lambda}^{2}\right\rangle_{4}^{\prime}}{\left\langle s_{\lambda^{\prime}}^{2}, s_{\lambda^{\prime}}^{2}\right\rangle_{4}^{\prime}} .
$$

It will suffice to show that the latter ratio is equal to 1 . Write $s_{\lambda}^{2}$ and $s_{\lambda^{\prime}}^{2}$ in the Schur basis:

$$
s_{\lambda}^{2}=\sum_{\nu} c_{\nu}^{\lambda} s_{\nu}, \quad s_{\lambda^{\prime}}^{2}=\sum_{\nu} c_{\nu}^{\lambda^{\prime}} s_{\nu}
$$

Since $\omega$ is an automorphism, for any partitions $\mu, \kappa$,

$$
\omega\left(s_{\mu} s_{\kappa}\right)=s_{\mu^{\prime}} s_{\kappa^{\prime}}
$$

In particular,

$$
s_{\lambda}^{2}=\omega \omega\left(s_{\lambda}^{2}\right)=\omega\left(s_{\lambda^{\prime}}^{2}\right) \Longrightarrow s_{\lambda^{\prime}}^{2}=\omega\left(s_{\lambda}^{2}\right)
$$

Comparing coefficients,

$$
\sum_{\nu} c_{\nu}^{\lambda^{\prime}} s_{\nu}=\sum_{\nu} c_{\nu}^{\lambda} s_{\nu^{\prime}}=\sum_{\nu^{\prime}} c_{\nu^{\prime}}^{\lambda} s_{\nu} \Longrightarrow c_{\nu}^{\lambda^{\prime}}=c_{\nu^{\prime}}^{\lambda}
$$

Expanding

$$
\left\langle s_{\lambda}^{2}, s_{\lambda}^{2}\right\rangle_{4}^{\prime}=\sum_{\nu_{1}, \nu_{2}} c_{\nu_{1}}^{\lambda} c_{\nu_{2}}^{\lambda}\left\langle s_{\nu_{1}}, s_{\nu_{2}}\right\rangle_{4}^{\prime}=\sum_{\nu_{1}, \nu_{2}} c_{\nu_{1}^{\prime}}^{\lambda} c_{\nu_{2}^{\prime}}^{\lambda}\left\langle s_{\nu_{1}^{\prime}}, s_{\nu_{2}^{\prime}}\right\rangle_{4}^{\prime}=\sum_{\nu_{1}, \nu_{2}} c_{\nu_{1}}^{\lambda^{\prime}} c_{\nu_{2}}^{\lambda^{\prime}}\left\langle s_{\nu_{1}^{\prime}}, s_{\nu_{2}^{\prime}}\right\rangle_{4}^{\prime}
$$




$$
=\sum_{\nu_{1}, \nu_{2}} c_{\nu_{1}}^{\lambda^{\prime}} c_{\nu_{2}}^{\lambda^{\prime}}\left\langle s_{\nu_{1}}, s_{\nu_{2}}\right\rangle_{4}^{\prime}=\left\langle s_{\lambda^{\prime}}^{2}, s_{\lambda^{\prime}}^{2}\right\rangle_{4}^{\prime}
$$

We have shown that conjugate partitions have asymptotically (assuming $\mathcal{N} \approx 1$ ) equal IPRs. This is not the case for finite $M$, when $\mathcal{N} \neq 1$. It would be interesting to give a physical explanation for this duality.

6.1. IPRs and diagonals of permutahedra. An alternate expression for $\sum_{\mathbf{x}}|c(\mathbf{x})|^{4}$ is obtained by multiplying out the terms of the determinant:

$$
\begin{aligned}
& \sum_{\mathbf{x}}|c(\mathbf{x})|^{4}=\frac{1}{M !} \sum_{P, Q, R, S \in S_{M}} \operatorname{sgn}(P Q R S) \prod_{j=1}^{M} \sum_{x_{1}, \ldots, x_{M}=1}^{L} e^{i x_{j}\left(k_{P(j)}+k_{Q(j)}-k_{R(j)}-k_{S(j)}\right)}= \\
= & \frac{L^{M}}{M !} \sum_{P, Q, R, S \in S_{M}} \operatorname{sgn}(P Q R S) \delta\left(k_{P(j)}+k_{Q(j)}-k_{R(j)}-k_{S(j)} \equiv 0 \quad \bmod 2 \pi, \forall j\right) .
\end{aligned}
$$

Using $k_{j}=\frac{2 \pi I_{j}}{L}$, this sum is equal to

$$
\begin{gathered}
\frac{L^{M}}{M !} \sum_{P, Q, R, S \in S_{M}} \operatorname{sgn}(P Q R S) \delta\left(I_{P(j)}+I_{Q(j)}-I_{R(j)}-I_{S(j)} \equiv 0 \quad \bmod L, \forall j\right) \\
\quad=\frac{L^{M}}{M !} \sum_{P, Q, R, S \in S_{M}} \operatorname{sgn}(P Q R S) \delta\left(I_{P}+I_{Q}-I_{R}-I_{S} \equiv 0 \quad \bmod L\right)
\end{gathered}
$$

Consequently,

$$
\mathbf{t}_{\lambda}=\frac{1}{L^{M} M !} \sum_{P, Q, R, S \in S_{M}} \operatorname{sgn}(P Q R S) \delta\left(I_{P}+I_{Q}-I_{R}-I_{S} \equiv 0 \quad \bmod L\right) .
$$

We may interpret the sum as follows. A permutahedron is the polytope obtained by taking the convex hull of all permutations of a fixed vector $\left(I_{1}, I_{2}, \ldots, I_{M}\right)$ with distinct entries. We can interpret $I_{P}-I_{R}$ as a diagonal vector of this permutahedron. Then a condition of the form

$$
I_{P}-I_{R}=I_{S}-I_{Q}
$$

would be a condition on two diagonals to be mutual translates and a congruence of the form

$$
I_{P}-I_{R} \equiv I_{S}-I_{Q} \quad(\bmod L)
$$

is interpreted similarly.

\section{Future Directions}

Several interesting questions remain to be studied.

1. Experimental results suggest that

$$
\lim _{M \rightarrow \infty} \frac{\left\langle J_{\lambda}^{(1 / 2)}, J_{\lambda}^{(1 / 2)}\right\rangle_{2 M ; 4}}{\left\langle J_{\lambda}^{(1 / 2)}, J_{\lambda}^{(1 / 2)}\right\rangle_{4}}=1 .
$$

In other words, for a finite number of variables, the discrete Hall inner product is asymptotically equal to the Hall inner product when $L=2 M$. The reason this is not a simple consequence of the convergence of a Riemann sum to the corresponding integral is that even though the number of sample points $(L)$ on each torus increases, the number of torii $(M)$ does as well. Nonetheless, experiment suggests that the error decreases (see Figure 10. If true, this will allow to extend the methods to compute the asymptotics of IPRs when $L=2 M$. 


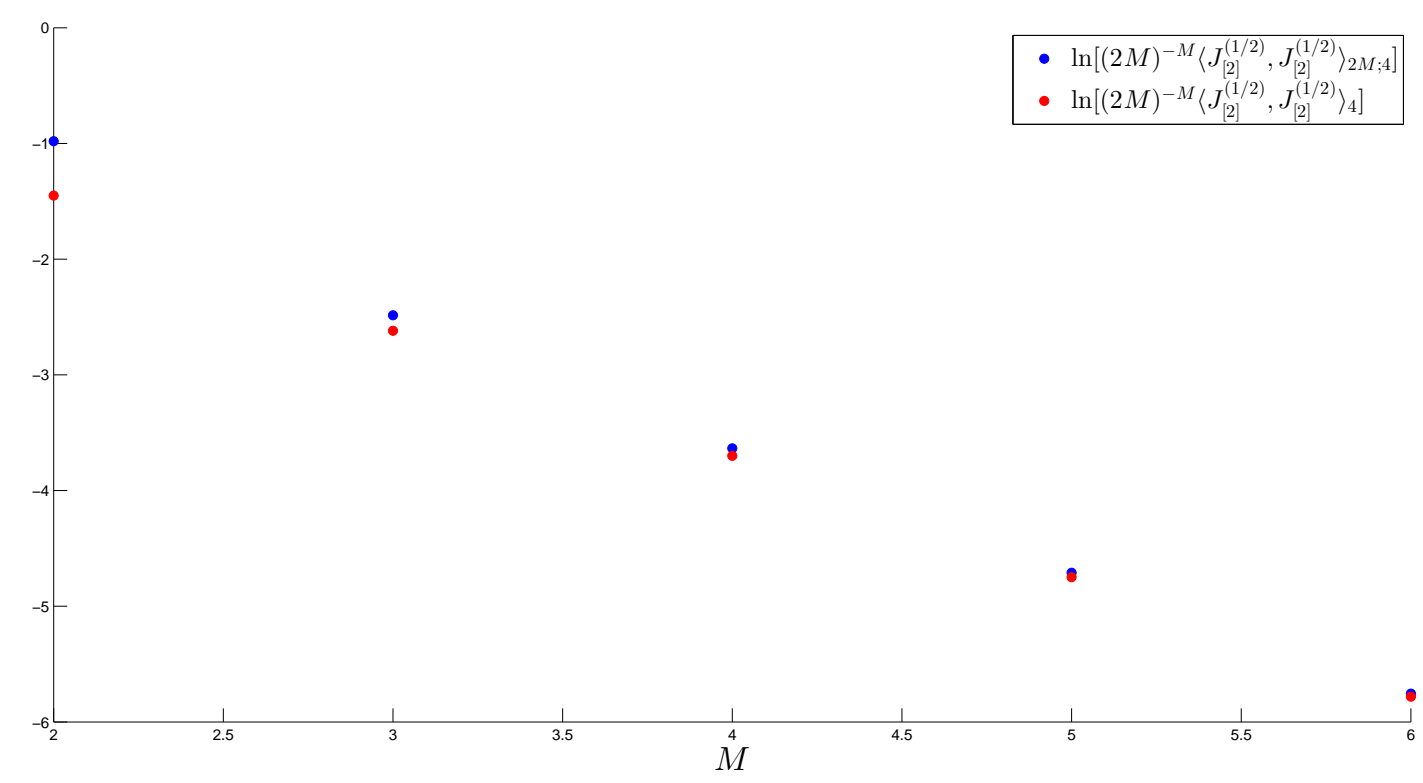

FIgURE 1. Plotted are the logs of $\frac{\left\langle J_{[2]}^{(1 / 2)}, J_{[2]}^{(1 / 2)}\right\rangle_{L ; 4}}{L^{M}}$ and $\frac{\left\langle J_{[2]}^{(1 / 2)}, J_{[2]}^{(1 / 2)}\right\rangle_{4}}{L^{M}}$ for $L=2 M$

2. There exists a dynamical interpretation of the IPRs (described in [MPL16]). In the case when the spectrum is nondegenerate, it is given by summing the IPRs:

$$
T:=\sum_{k} t_{k}=\sum_{i, k=1}^{D}\left|\left\langle a_{i} \mid \psi_{k}\right\rangle\right|^{4}
$$

This quantity measures how much the eigenstates are localized in the preferential basis. It can range from $T_{\min }=1$ (the eigenstates are spread maximally over the whole basis) to $T_{\max }=D$ (each eigenstate matches a basis vector). The ratio $T / D$ measures the stationary return probability to an initial basis state, averaged over all the basis states. The minimum value is reached if the dynamics connects any initial basis state to all the other basis states. On the other hand, if it takes on the maximum value, then the system does not evolve if it is initialized from a basis state.

3. The scalar product $\langle\cdot, \cdot\rangle_{L ; \beta}$ is a discretization of the Hall inner product. Using the Gram-Schmidt orthonormalization procedure on the symmetric monomial functions, we may define an orthonormal basis which is triangular with respect to the monomial symmetric basis. Such "discrete Jack polynomials" may have interesting properties.

4. The data table suggests that the IPR of the ground state is largest. The next few excited states, labeling by partitions, have IPRs which are approximately $1 / 2,1 / 3,1 / 4$ and $1 / 5$ of the ground state IPR, for $|\lambda|=1,2,3,4$. It is unlikely that this pattern will continue. It would be interesting to determine the distribution of the values of the IPRs.

Acknowledgments. The author would like to thank Vir Bulchandani, Mark Haiman, Joel Moore and Lauren Williams for their helpful comments in the writing of this manuscript. 


\section{REFERENCES}

[DES07] Ioana Dumitriu, Alan Edelman, and Gene Shuman. MOPS: multivariate orthogonal polynomials (symbolically). J. Symbolic Comput., 42(6):587-620, 2007.

[Dys62] Freeman J Dyson. Statistical theory of the energy levels of complex systems. i. Journal of Mathematical Physics, 3(1):140-156, 1962.

[ET72] JT Edwards and DJ Thouless. Numerical studies of localization in disordered systems. Journal of Physics C: Solid State Physics, 5(8):807, 1972.

[fWY11] Jian feng Wu and Ming Yu. Calogero-sutherland model in interacting fermion picture and explicit construction of jack states, 2011.

[Gau73] M Gaudin. Gaz coulombien discret à une dimension. Journal de Physique, 34(7):511-522, 1973.

[Gom14] Thomas Gomez. The boson-fermion correspondence: Formulation, proof and application to jack polynomials. Undergraduate thesis, Utrecht University, 2014.

[JM15] Tiefeng Jiang and Sho Matsumoto. Moments of traces of circular beta-ensembles. Ann. Probab., 43(6):3279-3336, 2015.

[Mac15] I. G. Macdonald. Symmetric functions and Hall polynomials. Oxford Classic Texts in the Physical Sciences. The Clarendon Press, Oxford University Press, New York, second edition, 2015. With contribution by A. V. Zelevinsky and a foreword by Richard Stanley, Reprint of the 2008 paperback edition [ MR1354144].

[MPL16] Grégoire Misguich, Vincent Pasquier, and Jean-Marc Luck. Inverse participation ratios in the xxz spin chain. Physical Review B, 94(15):155110, 2016.

[ŠB13] Ladislav Šamaj and Zoltán Bajnok. Introduction to the statistical physics of integrable many-body systems. Cambridge University Press, 2013.

[Sta99] Richard P. Stanley. Enumerative combinatorics. Vol. 2, volume 62 of Cambridge Studies in Advanced Mathematics. Cambridge University Press, Cambridge, 1999. With a foreword by Gian-Carlo Rota and appendix 1 by Sergey Fomin.

Department of Mathematics, University of California, Berkeley, CA 94720-3840

E-mail address: e.tsukerman@berkeley.edu 